\title{
Performance Analysis and Optimization for Cognitive Radio Networks with Classified Secondary Users and Impatient Packets
}

\author{
Yuan Zhao ${ }^{1,2}$ and Luyi Bai ${ }^{1,2}$ \\ ${ }^{1}$ School of Computer Science and Engineering, Northeastern University, Shenyang 110819, China \\ ${ }^{2}$ School of Computer and Communication Engineering, Northeastern University at Qinhuangdao, Qinhuangdao 066004, China \\ Correspondence should be addressed to Yuan Zhao; yuanzh85@163.com
}

Received 13 March 2017; Accepted 17 May 2017; Published 10 July 2017

Academic Editor: Alessandro Bazzi

Copyright ( 2017 Yuan Zhao and Luyi Bai. This is an open access article distributed under the Creative Commons Attribution License, which permits unrestricted use, distribution, and reproduction in any medium, provided the original work is properly cited.

\begin{abstract}
A cognitive radio network with classified Secondary Users (SUs) is considered. There are two types of SU packets, namely, SU1 packets and SU2 packets, in the system. The SU1 packets have higher priority than the SU2 packets. Considering the diversity of the SU packets and the real-time need of the interrupted SU packets, a novel spectrum allocation strategy with classified SUs and impatient packets is proposed. Based on the number of PU packets, SU1 packets, and SU2 packets in the system, by modeling the queue dynamics of the networks users as a three-dimensional discrete-time Markov chain, the transition probability matrix of the Markov chain is given. Then with the steady-state analysis, some important performance measures of the SU2 packets are derived to show the system performance with numerical results. Specially, in order to optimize the system actions of the SU2 packets, the individually optimal strategy and the socially optimal strategy for the SU2 packets are demonstrated. Finally, a pricing mechanism is provided to oblige the SU2 packets to follow the socially optimal strategy.
\end{abstract}

\section{Introduction}

Nowadays, spectrum crisis caused by possible shortage of wireless spectrum has attracted great attention of relevant scholars. However, some researches indicated that the utilization of some spectrum was not more that 6\% [1-3]. This highlights a paradox between the higher transmission request and the actual lower spectrum utilization. In order to solve the spectrum crisis, it is necessary to make effort to enhance the spectrum utilization. As a technology to enhance the spectrum utilization effectively, cognitive radio emerged. Following the technology of cognitive radio, cognitive radio networks begin to cause wide public concern [4-6].

In general cognitive radio networks, there are two types of users, namely, Primary Users (PUs) and Secondary Users (SUs). The SUs are allowed to use the spectrum licensed to the PUs when the spectrum is not occupied. This kind of opportunistic spectrum occupying way can reduce the spectrum holes effectively $[7,8]$.

This paper focuses on the spectrum allocation strategy in cognitive radio networks with classified SU packets and impatient interrupted SU packets. The system performance of the proposed cognitive radio networks is evaluated with a discrete-time Markov chain model to give the performance analysis of the system model. With the steady-state analysis, some performance measures of the SU2 packets are derived and the system access actions of the SU2 packets are optimized.

The remainder of this paper is organized as follows. Some related works are listed in Section 2. The system model and model analysis are demonstrated in Section 3. In Section 4, some performance measures of the SU2 packets are derived. In Section 5, the individually optimal strategy and the socially optimal strategy are given, and a pricing mechanism is also given. The numerical results are shown in Section 6. Finally, conclusions are drawn in Section 7.

\section{Related Works}

In recent years, the researches about cognitive radio networks begin to become a hot research point [9-12]. In [13], in order 
to reduce the possible bad influence to the PU packets from larger number of SU packets, the authors proposed an adaptive access control scheme for the SU packets. By building a two-dimensional Markov chain, the authors derived the expressions of the throughput and the average delay of the SU packets. In [14], considering the availability of a channel dynamically varying in time in cognitive radio networks, the authors designed a channel-availability estimation strategy based on the features of mobile scenarios. With numerical results, they showed and analyzed the channel availability among different mobile scenarios. In [15], by introducing a pricing policy for the SU packets, the authors proposed a novel energy saving strategy in cognitive radio networks. With a Quasi-Birth-and-Death (QBD) process model, the authors derived the expressions of the average latency of SU packets and the energy saving ratio. They also optimized the system actions of the SU packets.

From the researches mentioned above, it is not difficult to find that the papers were all studied in the cognitive radio networks with single type of SUs. However, there are different types of transmission requests in modern communication networks. It is also necessary to classify the SU packets with different requests in the research of cognitive radio networks. In $[16,17]$, the classification to the SU packets was considered. The authors assumed that there were two kinds of SU packets in the system, namely, SU1 packets and SU2 packets. They assumed that the SU1 packets had higher priority than the SU2 packets. With mathematical analysis, different performance measures, such as the throughput and the interrupted rate for the two types of SU packets, were derived, respectively.

On the other hand, as mentioned above, in most of researches in cognitive radio networks, the interrupted SU packets were assumed to wait in the system for following transmission. However, in practice networks, timeliness is very important to some kind of SU packets, especially to the interrupted packets. The interrupted SU packets may not wait in the system with enough patience. These packets can be called impatient packets in this paper. In order to guarantee the transmission continuity and reliability, the impatient interrupted packets may leave the system and hand off to another available spectrum for retransmission [18]. Therefore, it is necessary to consider these impatient interrupted packets when designing the spectrum allocation strategy in cognitive radio networks.

Moreover, in the researches of cognitive radio networks, queueing theory $[19,20]$ is an important tool to model the system when evaluating the performance of cognitive radio networks [21, 22]. In [21], a cognitive radio network with multiple channels and multiple users was considered. By modeling the queue dynamics as a discrete-time finitestate Markov chain, some performance measures, including average queueing delay, packet-loss rate, and effective throughput, were obtained. In [23], a priority-based continuous queueing model was built and analyzed to evaluate the system performance of a cognitive radio network with channel assembling. In [24], a cognitive radio network with multiple SUs were considered. Based on the queueing actions of the network users, a three-dimensional Markov chain was built to analyze the system performance. This paper is substantially different from [24] in that the impatient interrupted SU packets are considered in this paper to satisfy real-time need of the interrupted SU packets. On the other hand, with the assumption of the impatient interrupted SU packets, the transition probability matrix built in this paper is very different from [24] and the steady-state distribution is obtained by using the matrix-geometric solution method. Moreover, different from the threshold optimization in [24], the idea of game theory is introduced in this paper to optimize the system actions of the SU2 packets.

\section{System Model}

3.1. Model Assumption. In this paper, a cognitive radio network with a single channel is considered. There are two kinds of SU packets, namely, SU1 packets and SU2 packets in the system. The SU1 packets have higher priority than the SU2 packets. The PU packets in the system have the highest priority to occupy the single channel. Moreover, the spectrum sensing for the SUs is assumed to be ideal in this paper. It means that the interferences among different SUs are not considered in following system model.

There is a buffer (called SU2 buffer) set for the SU2 packets. On the arrival instant of an SU2 packet, if the channel is being occupied, this newly arriving SU2 packet will wait and queue in the SU2 buffer for later transmission. There are no buffer settings for the PU packets and the SU1 packets.

Considering the highest priority of the PU packets, when a PU packet appears during the middle of the transmission of an SU packet (an SU1 packet or an SU2 packet), the transmission for this SU packet will be interrupted directly.

Considering the relative priority of the SU1 packets, a newly arriving SU1 packet can only interrupt the transmission of an SU2 packet.

Considering the higher transmission continuity need of the interrupted packet, the interrupted SU1 packets and the interrupted SU2 packets are both impatient. Any interrupted SU packet (an SU1 packet or an SU2 packet) will not wait in the system for later transmission. These interrupted SU packets will choose to leave the system and hand off to another available channel for transmission.

Based on the spectrum allocation strategy mentioned above, considering the digital nature of modern networks, a discrete-time Markov chain model can be built as follows.

The time axis can be divided into slots with equal size, and the slot boundaries are denoted as $t=1,2, \ldots$. The arrival intervals of the PU packets, the SU1 packets, and the SU2 packets are assumed to follow geometric distributions with arrival rates $\lambda_{1}, \lambda_{21}$, and $\lambda_{22}$. The transmission times of the PU packets, the SU1 packets, and the SU2 packets are assumed to follow geometric distributions with service rates $\mu_{1}, \mu_{21}$, and $\mu_{22}$.

Let $S_{n}^{(2)}, S_{n}^{(1)}$, and $P_{n}$ be the number of SU2 packets, SU1 packets, and PU packets in the system at the instant $t=$ $n^{+}$, respectively. In order to describe the transitions of the number of different types of packets in the system, a threedimensional process composed of the number $S_{n}^{(2)}$ of the SU2 
packets, the number $S_{n}^{(1)}$ of the SU1 packets, and the number $P_{n}$ of the PU packets can be abstracted. Then $\left\{S_{n}^{(2)}, S_{n}^{(1)}, P_{n}\right\}$ constitutes a three-dimensional discrete-time Markov chain [20]. With the model assumption mentioned above, the state space $\Omega$ of $\left\{S_{n}^{(2)}, S_{n}^{(1)}, P_{n}\right\}$ can be given as follows:

$$
\Omega=\{(i, 0,0) \cup(i, 0,1) \cup(i, 1,0): 0 \leq i \leq \infty\} .
$$

3.2. Model Analysis. Let $\mathbf{P}$ be the state transition probability matrix of the three-dimensional Markov chain $\left\{S_{n}^{(2)}, S_{n}^{(1)}, P_{n}\right\}$. In the system model, the buffer capacity of the SU2 packets is assumed to be infinite to accommodate more SU2 packets in the system. Therefore, according to the state transition of the number of SU2 packets, the dimension of the state transition probability matrix $\mathbf{P}$ is infinite. This assumption is the same as that in [15]. $\mathbf{P}$ can be given as follows:

$$
\mathbf{P}=\left(\begin{array}{ccccc}
\mathbf{F}_{0} & \mathbf{I}_{0} & & & \\
\mathbf{D} & \mathbf{F} & \mathbf{I} & & \\
& \mathbf{D} & \mathbf{F} & \mathbf{I} & \\
& & \ddots & \ddots & \ddots
\end{array}\right) .
$$

Each nonzero block in $\mathbf{P}$ is a $3 \times 3$ matrix and can be given as follows:
(1) $F_{0}$ is the transition probability matrix which denotes the number of SU2 packets in the system being fixed at 0 :

$\mathbf{F}_{0}$

$=\left(\begin{array}{ccc}\bar{\lambda}_{1} \bar{\lambda}_{21} \bar{\lambda}_{22} & \lambda_{1} \bar{\lambda}_{22} & \bar{\lambda}_{1} \lambda_{21} \bar{\lambda}_{22} \\ \mu_{1} \bar{\lambda}_{1} \bar{\lambda}_{21} \bar{\lambda}_{22} & \left(\bar{\mu}_{1}+\lambda_{1} \mu_{1}\right) \bar{\lambda}_{22} & \mu_{1} \bar{\lambda}_{1} \lambda_{21} \bar{\lambda}_{22} \\ \mu_{21} \bar{\lambda}_{1} \bar{\lambda}_{21} \bar{\lambda}_{22} & \lambda_{1} \bar{\lambda}_{22} & \bar{\lambda}_{1} \bar{\lambda}_{22}\left(\bar{\mu}_{21}+\lambda_{21} \mu_{21}\right)\end{array}\right)$.

(2) $\mathbf{I}_{0}$ is the transition probability matrix which denotes the number of SU2 packets in the system being increased from 0 to 1 :

$\mathbf{I}_{0}$

$=\left(\begin{array}{ccc}\bar{\lambda}_{1} \bar{\lambda}_{21} \lambda_{22} & \lambda_{1} \lambda_{22} & \bar{\lambda}_{1} \lambda_{21} \lambda_{22} \\ \mu_{1} \bar{\lambda}_{1} \bar{\lambda}_{21} \lambda_{22} & \left(\bar{\mu}_{1}+\lambda_{1} \mu_{1}\right) \lambda_{22} & \mu_{1} \bar{\lambda}_{1} \lambda_{21} \lambda_{22} \\ \mu_{21} \bar{\lambda}_{1} \bar{\lambda}_{21} \lambda_{22} & \lambda_{1} \lambda_{22} & \bar{\lambda}_{1} \lambda_{22}\left(\bar{\mu}_{21}+\lambda_{21} \mu_{21}\right)\end{array}\right)$.

(3) D is the transition probability matrix which denotes the number of SU2 packets in the system being decreased by one:

$$
\mathbf{D}=\left(\begin{array}{ccc}
\mu_{22} \bar{\lambda}_{1} \bar{\lambda}_{21} \bar{\lambda}_{22} & \lambda_{1} \bar{\lambda}_{22} & \bar{\lambda}_{1} \lambda_{21} \bar{\lambda}_{22} \\
0 & 0 & 0 \\
0 & 0 & 0
\end{array}\right)
$$

(4) $\mathrm{F}$ is the transition probability matrix which denotes the number of SU2 packets in the system being fixed:

$$
\mathbf{F}=\left(\begin{array}{ccc}
\bar{\lambda}_{1} \bar{\lambda}_{21}\left(\lambda_{22} \mu_{22}+\bar{\lambda}_{22} \bar{\mu}_{22}\right) & \lambda_{1} \lambda_{22} & \bar{\lambda}_{1} \lambda_{21} \lambda_{22} \\
\mu_{1} \bar{\lambda}_{22} \bar{\lambda}_{1} \bar{\lambda}_{21} & \left(\bar{\mu}_{1}+\lambda_{1} \mu_{1}\right) \bar{\lambda}_{22} & \bar{\lambda}_{22} \bar{\lambda}_{1} \lambda_{21} \mu_{1} \\
\mu_{21} \bar{\lambda}_{22} \bar{\lambda}_{1} \bar{\lambda}_{21} & \lambda_{1} \bar{\lambda}_{22} & \left(\bar{\mu}_{21}+\lambda_{21} \mu_{21}\right) \bar{\lambda}_{22} \bar{\lambda}_{1}
\end{array}\right) .
$$

(5) I is the transition probability matrix which denotes the number of SU2 packets in the system being increased by one:

I

$$
=\left(\begin{array}{ccc}
\bar{\mu}_{22} \bar{\lambda}_{1} \bar{\lambda}_{21} \lambda_{22} & 0 & 0 \\
\mu_{1} \bar{\lambda}_{1} \bar{\lambda}_{21} \lambda_{22} & \left(\bar{\mu}_{1}+\lambda_{1} \mu_{1}\right) \lambda_{22} & \mu_{1} \bar{\lambda}_{1} \lambda_{21} \lambda_{22} \\
\mu_{21} \bar{\lambda}_{1} \bar{\lambda}_{21} \lambda_{22} & \lambda_{1} \lambda_{22} & \bar{\lambda}_{1} \lambda_{22}\left(\bar{\mu}_{21}+\lambda_{21} \mu_{21}\right)
\end{array}\right) .
$$

Until now, all the nonzero matrices in $\mathbf{P}$ have been given. Based on the structure of the transition probability matrix $\mathbf{P}$, the three-dimensional Markov chain $\left\{S_{n}^{(2)}, S_{n}^{(1)}, P_{n}\right\}$ follows a Quasi-Birth-and-Death (QBD) process [25]. The steady-state distribution $\pi_{i, j, k}$ of the three-dimensional Markov chain $\left\{S_{n}^{(2)}, S_{n}^{(1)}, P_{n}\right\}$ is defined as follows:

$$
\pi_{i, j, k}=\lim _{n \rightarrow \infty} P\left\{S_{n}^{(2)}=i, S_{n}^{(1)}=j, P_{n}=k\right\} .
$$

The numerical results for the steady-state distribution $\pi_{i, j, k}$ can be obtained by using the matrix-geometric solution method [26].

\section{Performance Measures of the SU2 Packets}

In the considered cognitive radio network with classified Secondary Users and impatient packets, the transmissions of the SU1 packets are influenced only by the PU packets and independent of the SU2 packets. The transmission processes of the PU packets and the SU1 packets can be regarded as a simple pure losing priority queueing model with single server. 
The system performance of the SU1 packets can reference the analysis results in [24].

On the other hand, in this paper, the transmissions of the SU2 packets are influenced not only by the PU packets but also by the SU1 packets. Therefore, this section only focuses on analyzing the performance measures of the SU2 packets. The expressions of some important performance measures, such as the interrupted rate, the throughput, and the average delay of the SU2 packets are derived.

The interrupted rate $\gamma$ of the SU2 packets is defined as the number of SU2 packets that are interrupted and leave the system per slot. The expression of the interrupted rate $\gamma$ of the SU2 packets can be given as follows:

$$
\gamma=\sum_{i=1}^{\infty} \pi_{i, 0,0} \bar{\mu}_{22}\left(1-\bar{\lambda}_{1} \bar{\lambda}_{21}\right) .
$$

The throughput $\theta$ of the SU2 packets is defined as the number of SU2 packets that are transmitted successfully per slot. An SU2 packet can be transmitted successfully if and only if the transmission of this SU2 packet is not be interrupted. The expression of the throughput $\theta$ of the SU2 packets can be given as follows:

$$
\theta=\lambda_{22}-\gamma .
$$

The average delay $\delta$ of the SU2 packets is defined as the average time length from an SU2 packet arriving at the system to this SU2 packet leaving the system (being transmitted successfully or being interrupted to leave). By using Little's formula [25], the expression of the average delay $\delta$ of the SU2 packets can be given as follows:

$$
\delta=\frac{E[\mathrm{SU} 2]}{\lambda_{22}},
$$

where $E[S U 2]$ is the average number of SU2 packets in the system in steady state. The expression of $E[\mathrm{SU} 2]$ can be given as follows:

$$
E[\mathrm{SU} 2]=\sum_{i=0}^{\infty} i\left(\pi_{i, 0,0}+\pi_{i, 0,1}+\pi_{i, 1,0}\right) .
$$

\section{Performance Optimization for the SU2 Packets}

In the cognitive radio networks with classified Secondary Users and impatient packets, the transmission of an SU packet that chooses to join the system may be interrupted by a PU packet or an SU1 packet. It means that an SU2 packet may not obtain a successful transmission after accessing the system. Therefore, for a newly arriving SU2 packet, it is necessary to weigh whether or not to join the system. This section focuses on optimizing the system access actions of the SU2 packets.

5.1. Individual and Social Optimization. Firstly, the system access action of a single SU2 packet should be analyzed. In following analysis, it is assumed that a newly arriving
SU2 packet cannot know the number of packets (including PU packets, SU1 packets, and SU2 packets) in the system before making the system access decision. If an SU2 packet is transmitted successfully, a reward denoted as $R$ can be received, while an SU2 packet that chooses to join the system has to bear a cost denoted as $C$ per slot. For a single SU2 packet, if this SU2 packet chooses to join the system, the individual net benefit function $W_{I}\left(\lambda_{22}\right)$ for this SU2 packet can been given as follows:

$$
W_{I}\left(\lambda_{22}\right)=\left(1-\frac{\gamma}{\lambda_{22}}\right) R-\delta C .
$$

In (13), $1-\gamma / \lambda_{22}$ denotes the probability that an SU2 packet can obtain a successful transmission.

Let $\lambda_{e}$ be the individually optimal access rate of the SU2 packets and $q_{e}$ be the individually optimal access probability of the SU2 packets. Let $\Lambda$ be the potential arrival rate of the SU2 packets. Then $\lambda_{e}=q_{e} \Lambda$. As will be seen in following numerical results, the individual net benefit function will show a decreasing change trend as the arrival rate of the SU2 packets increases. The individually optimal strategy for the SU2 packets can be given as follows (to avoid a trivial solution, it is assumed that $\left.W_{I}\left(0^{+}\right)>0\right)$ :

(1) For the case of $W_{I}(\Lambda) \geq 0$, the individually optimal access probability is $q_{e}=1$, and the individually optimal access rate is $\lambda_{e}=q_{e} \Lambda=\Lambda$.

(2) For the case of $W_{I}(\Lambda)<0$, based on the Nash equilibrium theory [27], the individually optimal access rate can be obtained by solving $W_{I}\left(\lambda_{22}\right)=0$, and then the individually optimal access probability can be given by $q_{e}=\lambda_{e} / \Lambda$.

Secondly, the socially optimal strategy for the SU2 packets should be analyzed. The socially optimal strategy is to maximize the expected benefit received per slot. Therefore, the social net benefit function $W_{S}\left(\lambda_{22}\right)$ for the SU2 packet can be given as follows:

$$
W_{S}\left(\lambda_{22}\right)=\lambda_{22}\left(\left(1-\frac{\gamma}{\lambda_{22}}\right) R-\delta C\right) .
$$

Let $\lambda_{s}$ be the socially optimal access rate of the SU2 packets and $q_{s}$ be the socially optimal access probability of the SU2 packets. Then $\lambda_{s}=q_{s} \Lambda$. From (14), the socially optimal access rate $\lambda_{s}$ can be derived as follows:

$$
\lambda_{s}=\underset{\lambda_{22}}{\arg \max }\left\{W_{S}\left(\lambda_{22}\right)\right\} .
$$

With the socially optimal access rate $\lambda_{s}$ in (15), the socially optimal access probability $q_{s}=\lambda_{s} / \Lambda$ can be further obtained.

5.2. Pricing Mechanism. Just as will be seen in the numerical results, the optimal access rate and optimal access probability in the individually optimal strategy are higher than that in the socially optimal strategy. This means that more SU2 packets will choose to access the system in the individually optimal strategy, but this will reduce the social net benefit. In order 
to guarantee the maximum of the social net benefit, it is necessary to oblige the SU2 packets to follow the socially optimal access rate and probability. Therefore, an extra price $f$ is introduced to the SU2 packets with successful transmission. When an SU2 packet is transmitted successfully, this SU2 packet has to pay an extra price $f$. With $f$, the new individual net benefit function $\widehat{W}_{I}\left(\lambda_{22}\right)$ for a single SU2 packet can be given as follows:

$$
\widehat{W}_{I}\left(\lambda_{22}\right)=\left(1-\frac{\gamma}{\lambda_{22}}\right)(R-f)-\delta C .
$$

When an extra price $f$ is imposed, the new social net benefit function $\widehat{W}_{S}\left(\lambda_{22}\right)$ can be given as follows:

$$
\begin{aligned}
\widehat{W}_{S}\left(\lambda_{22}\right)= & \lambda_{22}\left(\left(1-\frac{\gamma}{\lambda_{22}}\right)(R-f)-\delta C\right) \\
& +\lambda_{22}\left(1-\frac{\gamma}{\lambda_{22}}\right) f .
\end{aligned}
$$

By comparing (14) and (17), it can be found that the extra price does not influence the social net benefit. This conclusion is the same as that in $[13,28,29]$. Based on the Nash equilibrium theory [28], by setting $\lambda_{22}=\lambda_{s}$ in (16), $\widehat{W}_{I}\left(\lambda_{s}\right)$ can be given as

$$
\widehat{W}_{I}\left(\lambda_{s}\right)=\left(1-\frac{\gamma}{\lambda_{s}}\right)(R-f)-\delta C .
$$

By solving $\widehat{W}_{I}\left(\lambda_{s}\right)=0$, the expression of the optimal extra price $f$ that realizes the maximum social net benefit can be given as follows:

$$
f=R-\frac{\lambda_{s} \delta C}{\lambda_{s}-\gamma} .
$$

Moreover, when the socially optimal access rate $\lambda_{s}$ is equal to the potential arrival rete $\Lambda$, the optimal extra price will be equal to or less than $f^{*}$, which can be given as follows:

$$
f^{*}=R-\frac{\Lambda \delta C}{\Lambda-\gamma} .
$$

\section{Numerical Results}

6.1. Numerical Results for the Performance Measures. In this subsection, the system performance of the SU2 packets is evaluated with numerical results. The throughput and the average delay are the most important performance measurers when evaluating the system performance of cognitive radio networks. In following numerical results, the change trends of the throughput and the average delay of the SU2 packets with different parameter settings are focused on. By referencing the parameter settings in [13], without loss of generality, the service rates $\mu_{1}, \mu_{21}$, and $\mu_{22}$ are assumed to be equal to 0.5 .

Figure 1 shows the change trend for the throughput of the SU2 packets with different arrival rates of the PU packets.

From Figure 1, it can be found that as the arrival rate $\lambda_{1}$ of the PU packets or the arrival rate $\lambda_{21}$ of the SU1 packets

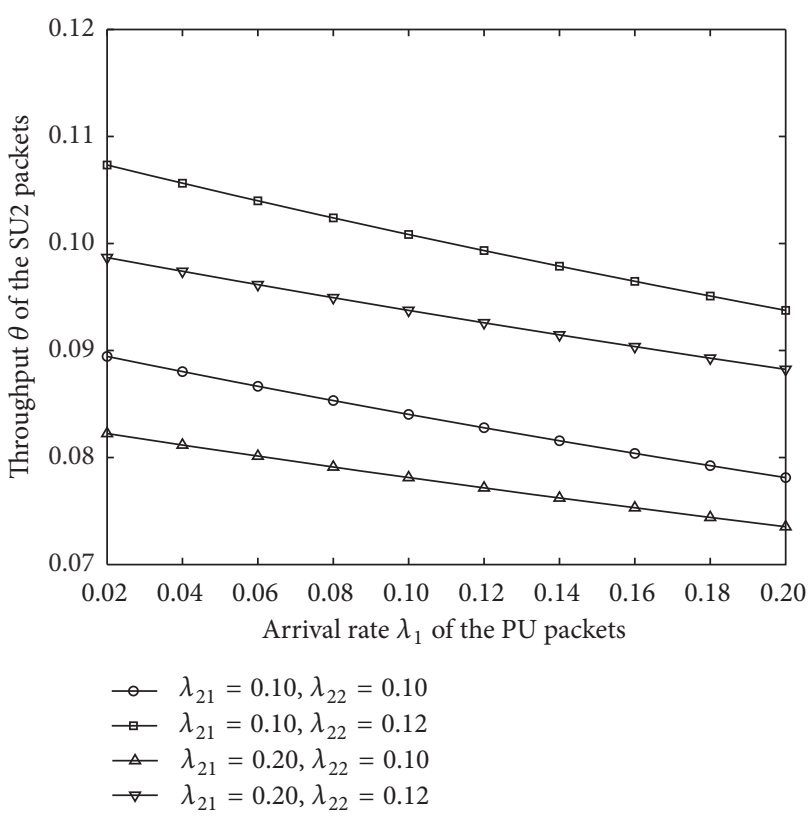

FIGURE 1: Change trend for the throughput $\theta$ of the SU2 packets.

increases, the throughput $\theta$ of the SU2 packets will decrease. The reason is that as the arrival rate of the PU packets or the arrival rate of the SU1 packets increases, more PU packets or SU1 packets will join the system, and the possibility for the SU2 packets occupying the channel will decrease. As a result, the number of SU2 packets being transmitted successfully will decrease, and then the throughput of the SU2 packets will be lower.

On the other hand, from Figure 1, it can be also found that as the arrival rate $\lambda_{22}$ of the SU2 packets increases, the throughput $\theta$ of the SU2 packets will increase. The reason for this change trend is that as the arrival rate of the SU2 packets increases, more SU2 packets will join the system, and more SU2 packets will be transmitted successfully. As a result, the throughput of the SU2 packets will be increased.

Figure 2 shows the change trend for the average delay of the SU2 packets with different arrival rates of the PU packets. Moreover, in Figure 2, the scheme with impatient interrupted SU2 packets proposed in this paper is denoted as Scheme I, and the conventional scheme without considering impatient interrupted SU2 packets is denoted as Scheme II. Figure 2 also compares the average delay of the SU2 packets between the two schemes.

From Figure 2, it can be concluded that as the arrival rate $\lambda_{1}$ of the PU packets or the arrival rate $\lambda_{21}$ of the SU1 packets increases, the average delay $\delta$ of the SU2 packets will increase. This is because as the arrival rate of the PU packets or the arrival rate of the SU1 packets increases, more PU packets or SU1 packets will occupy the channel, and more SU2 packets have to wait in the buffer. As a result, the number of SU2 packets in the system will increase, and then the average delay of the SU2 packets will be longer.

On the other hand, from Figure 2, it can be concluded that as the arrival rate $\lambda_{22}$ of the SU2 packets increases, the 


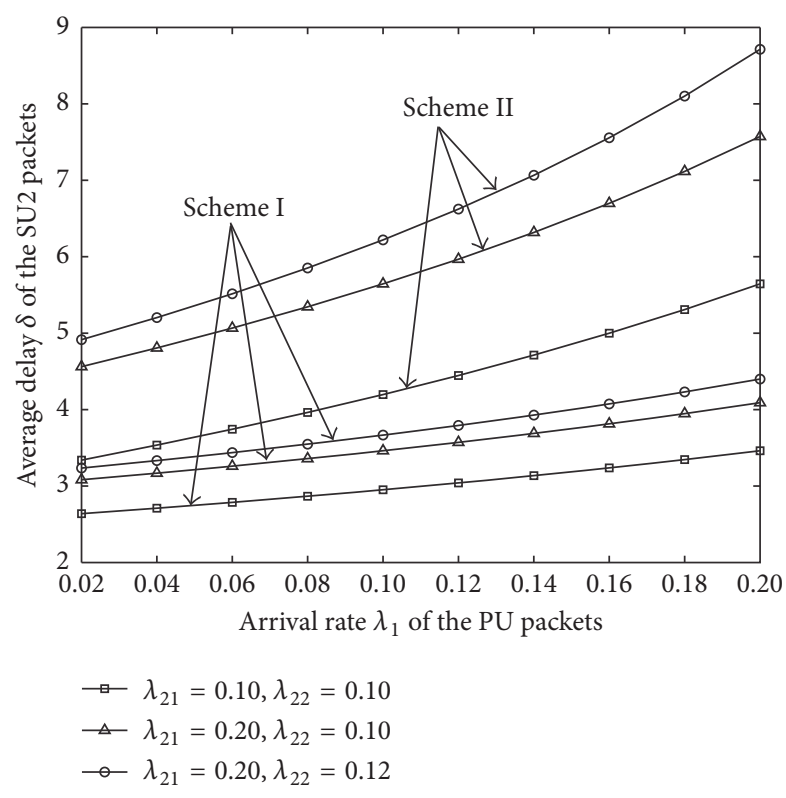

FIGURE 2: Change trend for the average delay $\delta$ of the SU2 packets.

average delay $\delta$ of the SU2 packets will increase. The reason may be that as the arrival rate of the SU2 packets increases, more SU2 packets will join the system, and more SU2 packets have to wait in the buffer. As a result, the average delay of the SU2 packets will be increased.

Moreover, from Figure 2, it can be found that, compared with the conventional scheme without considering impatient interrupted SU2 packets (Scheme II), the scheme with impatient interrupted SU2 packets proposed in this paper (Scheme I) realizes a shorter average delay of the SU2 packets. Therefore, it can be concluded that the scheme proposed in this paper can effectively reduce the average delay of the SU2 packets and is more suitable for the networks with a strict delay limit.

6.2. Numerical Results for the Optimal Strategies. In this subsection, the numerical results of the individually optimal strategy and the socially optimal strategy for the SU2 packets are compared. The numerical results for the extra price with different parameter settings are also shown. With some common parameters used in Section 6.1, by setting $C=2$, $\Lambda=0.2$ as an example, Figures 3 and 4 show the change trends for the individual net benefit function and the social net benefit function, respectively.

From Figures 3 and 4, it can be found that the individual net benefit function shows a decreasing tendency as the arrival rate $\lambda_{22}$ of the SU2 packets increases. On the other hand, the social net benefit function shows an upper convex tendency as the arrival rate $\lambda_{22}$ of the SU2 packets increases. Based on the analysis in Section 5.1 and the numerical results shown in Figures 3 and 4, the value range for the individually optimal strategy and the values for the socially optimal strategy with numerical results can be summarized in Table 1.

Firstly, because of the complexity of the individual net benefit function, it is not easy to give the exact value for the

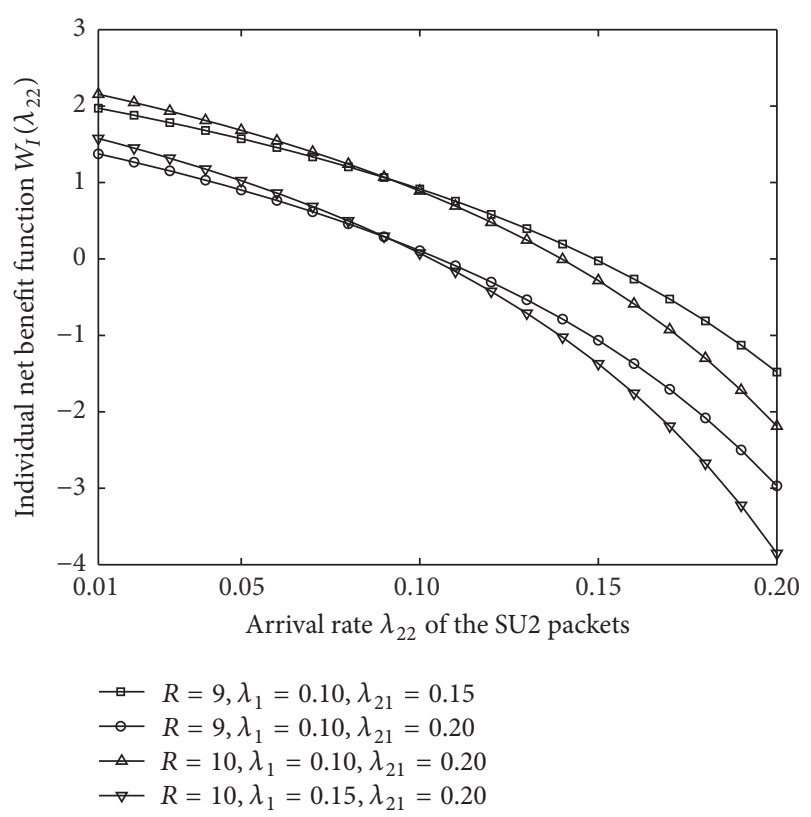

FIGURE 3: Change trend for the individual net benefit function $W_{I}\left(\lambda_{22}\right)$.

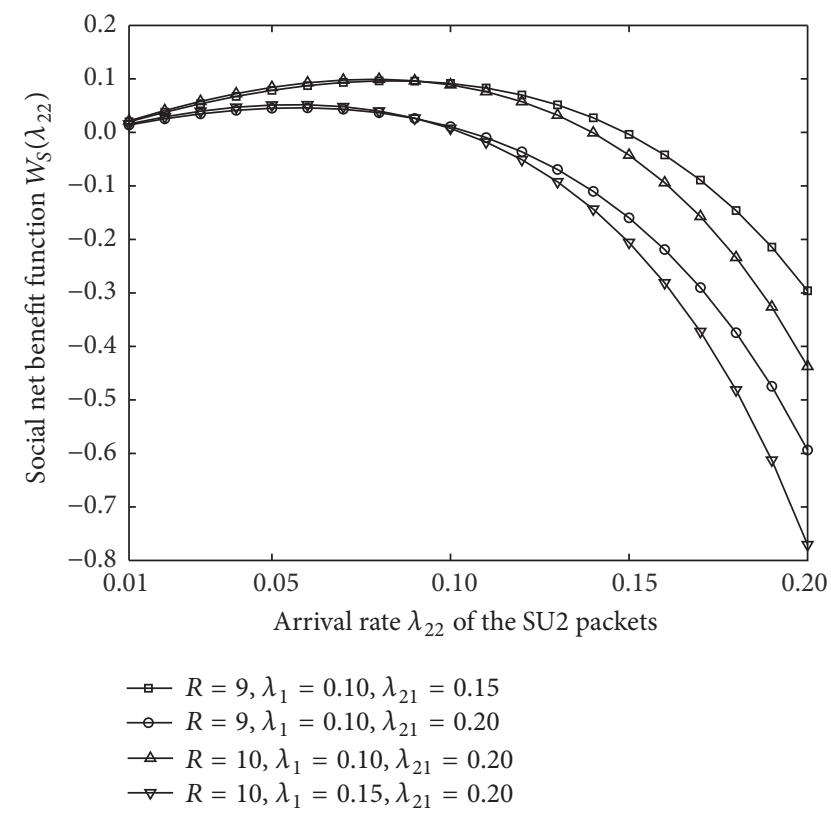

FIGURE 4: Change trend for the social net benefit function $W_{S}\left(\lambda_{22}\right)$.

individually optimal strategy. Therefore, the value ranges for the individually optimal strategy are summarized in Table 1, which does not affect the conclusion. From Table 1, it can be found that the optimal access rate and optimal access probability in the individually optimal strategy are higher than that in the socially optimal strategy. These conclusions are the same as that in [30].

On the other hand, from Table 1, it can be concluded that as the arrival rate $\lambda_{1}$ of the PU packets or the arrival rate $\lambda_{21}$ of the SU1 packets increases, the individually optimal access rate 
TABLE 1: Numerical results for the individually and socially optimal strategies.

\begin{tabular}{|c|c|c|c|c|c|c|c|c|}
\hline \multirow{2}{*}{$R$} & \multirow{2}{*}{$\lambda_{1}$} & \multirow{2}{*}{$\lambda_{21}$} & \multicolumn{2}{|c|}{$\lambda_{e}$} & \multicolumn{2}{|c|}{$q_{e}$} & \multirow{2}{*}{$\lambda^{*}$} & \multirow{2}{*}{$q^{*}$} \\
\hline & & & $\min$ & $\max$ & $\min$ & $\max$ & & \\
\hline 9 & 0.10 & 0.15 & 0.14 & 0.15 & 0.70 & 0.75 & 0.08 & 0.40 \\
\hline 9 & 0.10 & 0.20 & 0.10 & 0.11 & 0.50 & 0.55 & 0.06 & 0.30 \\
\hline 10 & 0.10 & 0.20 & 0.13 & 0.14 & 0.65 & 0.70 & 0.08 & 0.40 \\
\hline 10 & 0.15 & 0.20 & 0.10 & 0.11 & 0.50 & 0.55 & 0.06 & 0.30 \\
\hline
\end{tabular}

TABLE 2: Numerical results for the optimal extra price.

\begin{tabular}{lcccc}
\hline$R$ & $\lambda_{1}$ & $\lambda_{21}$ & $\lambda^{*}$ & $f$ \\
\hline 9 & 0.10 & 0.15 & 0.08 & 1.4879 \\
9 & 0.10 & 0.20 & 0.06 & 0.9787 \\
10 & 0.10 & 0.20 & 0.08 & 1.5668 \\
10 & 0.15 & 0.20 & 0.06 & 1.1371 \\
\hline
\end{tabular}

(probability) and the socially optimal access rate (probability) decrease. This is because as the arrival rate of the PU packets or the arrival rate of the SU1 packets increases, the possibility for the SU2 packets being transmitted successfully will be lower. As a result, more SU2 packets will not choose to access the system.

Moreover, from Table 1, it can be found that as the reward $R$ increases, the individually optimal access rate (probability) and the socially optimal access rate (probability) increase. The reason for this tendency is obvious that the higher the reward is, the more the SU2 packets will choose to access the system driven by the greater interests.

With the numerical results shown in Table 1, the numerical results for the optimal extra price can be further summarized in Table 2.

From Table 2, it can be found that as the arrival rate $\lambda_{1}$ of the PU packets or the arrival rate $\lambda_{21}$ of the SU1 packets increases, the optimal extra price $f$ decreases. This is because as the arrival rate of the PU packets or the arrival rate of the SU1 packets increases, more SU2 packets will choose not to access the system. In order to encourage more SU2 packets to access the system, the extra price has to be reduced.

On the other hand, from Table 2, it can be found that as the reward $R$ increases, the optimal extra price $f$ increases. The reason for this tendency is that the higher the reward is, the more the SU2 packets will choose to access the system. In order to control the access of the SU2 packets, the extra price should be set higher.

\section{Conclusion}

This paper considers a cognitive radio network with classified Secondary Users and impatient packets. The interrupted SU1 packets and the interrupted SU2 packets would leave the system to find another available channel. Based on the system actions of the three types of packets, a discrete-time Markov chain was built and analyzed, and then the expressions of the interrupted rate, the throughput, and the average delay of the SU2 packets were derived to describe the system performance with numerical results. The numerical results showed that as the arrival rate of the SU2 packets increased, the average delay of the SU2 packets would increase obviously. Therefore, in order to control and further optimize the system access actions of the SU2 packets, an individually optimal strategy and a socially optimal strategy for the SU2 packets were given. A pricing mechanism was described to oblige the SU2 packets to adopt the socially optimal strategy. Moreover, the numerical results for the optimal strategies were showed and analyzed.

In this paper, a system model with ideal spectrum sensing among the SUs and an infinite buffer setting for the SU2 packets in a cognitive radio network was analyzed. As a future work, another system model should be analyzed by considering the interferences among different SUs and a finite buffer setting for the SU2 packets.

\section{Conflicts of Interest}

The authors declare that the funding mentioned in Acknowledgments does not lead to any conflicts of interest. Additionally, the authors declare that there are no conflicts of interest regarding the publication of this manuscript.

\section{Acknowledgments}

This work was supported by the Natural Science Foundation of Hebei Province (F2016501073 and F2015501049), the Fundamental Research Funds for the Central Universities (N152303007), the Doctoral Scientific Research Foundation of Liaoning Province (201601016), the National Natural Science Foundation of China (61472342 and 61402087), the Scientific Research Fund of Hebei Education Department (QN2016307), and the Doctoral Fund Project of Northeastern University at Qinhuangdao (XNB201606), China.

\section{References}

[1] Q. Zhao and B. M. Sadler, "A survey of dynamic spectrum access," IEEE Signal Processing Magazine, vol. 24, no. 3, pp. 7989, 2007.

[2] B. Wang and K. J. R. Liu, "Advances in cognitive radio networks: a survey," IEEE Journal on Selected Topics in Signal Processing, vol. 5, no. 1, pp. 5-23, 2011.

[3] G. Ghosh, P. Das, and S. Chatterjee, "Cognitive radio and dynamic spectrum access-A study," International Journal of Next-Generation Networks, vol. 6, no. 1, pp. 43-60.

[4] J. Marinho and E. Monteiro, "Cognitive radio: survey on communication protocols, spectrum decision issues, and future 
research directions," Wireless Networks, vol. 18, no. 2, pp. 147164, 2012.

[5] E. Z. Tragos, S. Zeadally, A. G. Fragkiadakis, and V. A. Siris, "Spectrum assignment in cognitive radio networks: a comprehensive survey," IEEE Communications Surveys and Tutorials, vol. 15, no. 3, pp. 1108-1135, 2013.

[6] A. De Domenico, E. Calvanese Strinati, and M.-G. Di Benedetto, "A survey on MAC strategies for cognitive radio networks," IEEE Communications Surveys and Tutorials, vol. 14, no. 1, pp. 21-44, 2012.

[7] B. Awoyemi, B. T. J. Maharaj, and A. S. Alfa, "Solving resource allocation problems in cognitive radio networks: a survey," EURASIP Journal on Wireless Communications and Networking, vol. 2016, no. 1, article 176, 2016.

[8] B. Sabin and M. Sangman, "A survey of MAC protocols for cognitive radio body area networks," Sensors, vol. 15, no. 4, pp. 9189-9209, 2015.

[9] M. Naeem, A. Anpalagan, M. Jaseemuddin, and D. C. Lee, "Resource allocation techniques in cooperative cognitive radio networks," IEEE Communications Surveys and Tutorials, vol. 16, no. 2, pp. 729-744, 2014.

[10] B. Benmammar, A. Amraoui, and F. Krief, "A survey on dynamic spectrum access techniques in cognitive radio networks," International Journal of Communication Networks and Information Security, vol. 5, no. 2, pp. 68-79, 2013.

[11] C. Jiang, Y. Chen, K. J. R. Liu, and Y. Ren, "Optimal pricing strategy for operators in cognitive femtocell networks," IEEE Transactions on Wireless Communications, vol. 13, no. 9, pp. 5288-5301, 2014.

[12] C. Jiang, Y. Chen, Y.-H. Yang, C.-Y. Wang, and K. J. R. Liu, "Dynamic Chinese restaurant game: theory and application to cognitive radio networks," IEEE Transactions on Wireless Communications, vol. 13, no. 4, pp. 1960-1973, 2014.

[13] S. Jin, Y. Zhao, W. Yue, and Z. Saffer, "Performance analysis and optimization of an adaptive admission control scheme in cognitive radio networks," Mathematical Problems in Engineering, vol. 2013, Article ID 727310, 2013.

[14] A. S. Cacciapuoti, M. Caleffi, L. Paura, and M. A. Rahman, "Channel availability for mobile cognitive radio networks," Journal of Network and Computer Applications, vol. 47, pp. 131136, 2015.

[15] S. Jin, S. Chen, and J. Zhang, "Social optimization and pricing policy in cognitive radio networks with an energy saving strategy," Mobile Information Systems, vol. 2016, Article ID 2426580, 2016.

[16] Y. Lee, C. G. Park, and D. B. Sim, "Cognitive radio spectrum access with prioritized secondary users," Applied Mathematics \& Information Sciences, vol. 6, no. 2S, pp. 595S-601S, 2012.

[17] Y. Zhang, T. Jiang, L. Zhang, D. Qu, and W. Peng, "Analysis on the transmission delay of priority-based secondary users in cognitive radio networks," in Proceedings of the International Conference on Wireless Communications \& Signal Processing, 6 pages, IEEE Press, Hangzhou, China, 2013.

[18] I. Christian, S. Moh, I. Chung, and J. Lee, "Spectrum mobility in cognitive radio networks," IEEE Communications Magazine, vol. 50, no. 6, pp. 114-121, 2012.

[19] D. Gross, J. Shortle, J. M. Thompson, and C. M. Harris, Fundamentals of Queueing Theory, John Wiley \& Sons, Hoboken, NJ, USA, 4th edition, 2008.

[20] H. Takagi, "Queueing Analysis," in Discrete-Time Systems, vol. 3, North-Holland Elsevier, Amsterdam, The Netherlands, 1993.
[21] J. Wang, A. Huang, L. Cai, and W. Wang, "On the queue dynamics of multiuser multichannel cognitive radio networks," IEEE Transactions on Vehicular Technology, vol. 62, no. 3, pp. 1314-1328, 2013.

[22] C. Jiang, Y. Chen, K. J. Ray Liu, and Y. Ren, "Renewal-theoretical dynamic spectrum access in cognitive radio network with unknown primary behavior," IEEE Journal on Selected Areas in Communications, vol. 31, no. 3, pp. 406-416, 2013.

[23] I. A. M. Balapuwaduge, L. Jiao, V. Pla, and F. Y. Li, “Channel assembling with priority-based queues in cognitive radio networks: strategies and performance evaluation," IEEE Transactions on Wireless Communications, vol. 13, no. 2, pp. 630-645, 2014.

[24] Y. Zhao, J. Wang, J. Liu, and L. Bai, “Optimization of access threshold for cognitive radio networks with prioritized secondary users," Mobile Information Systems, vol. 2016, Article ID 3297938, 8 pages, 2016.

[25] N. Tian and Z. G. Zhang, Vacation Queueing Models: Theory and Applications, Springer, New York, NY, USA, 2006.

[26] A. S. Alfa, Queueing Theory for Telecommunication: Discrete Time Modelling of a Single Node System, Springer, New York, NY, USA, 2010.

[27] R. Hassin and M. Haviv, To Queue or Not to Queue: Equilibrium Behavior in Queueing Systems, Kluwer Academic, Boston, Mass, USA, 2003.

[28] R. Hassin, Rational Queueing, CRC Press, Boca Raton, FL, USA, 2016.

[29] C. T. Do, N. H. Tran, M. Van Nguyen, C. S. Hong, and S. Lee, "Social optimization strategy in unobserved queueing systems in cognitive radio networks," IEEE Communications Letters, vol. 16, no. 12, pp. 1944-1947, 2012.

[30] H. Li and Z. Han, "Socially optimal queuing control in cognitive radio networks subject to service interruptions: to queue or not to queue?" IEEE Transactions on Wireless Communications, vol. 10, no. 5, pp. 1656-1666, 2011. 

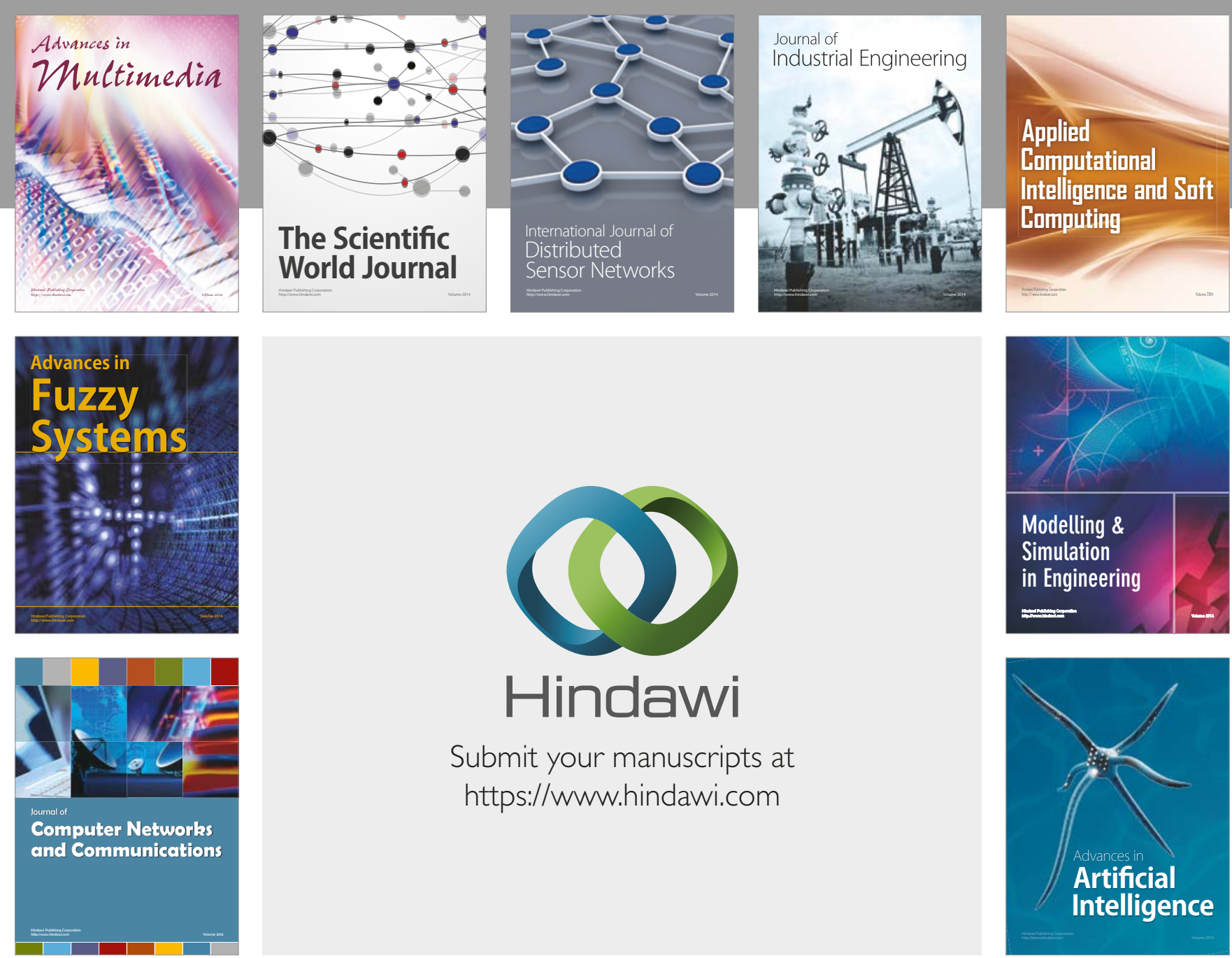

\section{Hindawi}

Submit your manuscripts at

https://www.hindawi.com
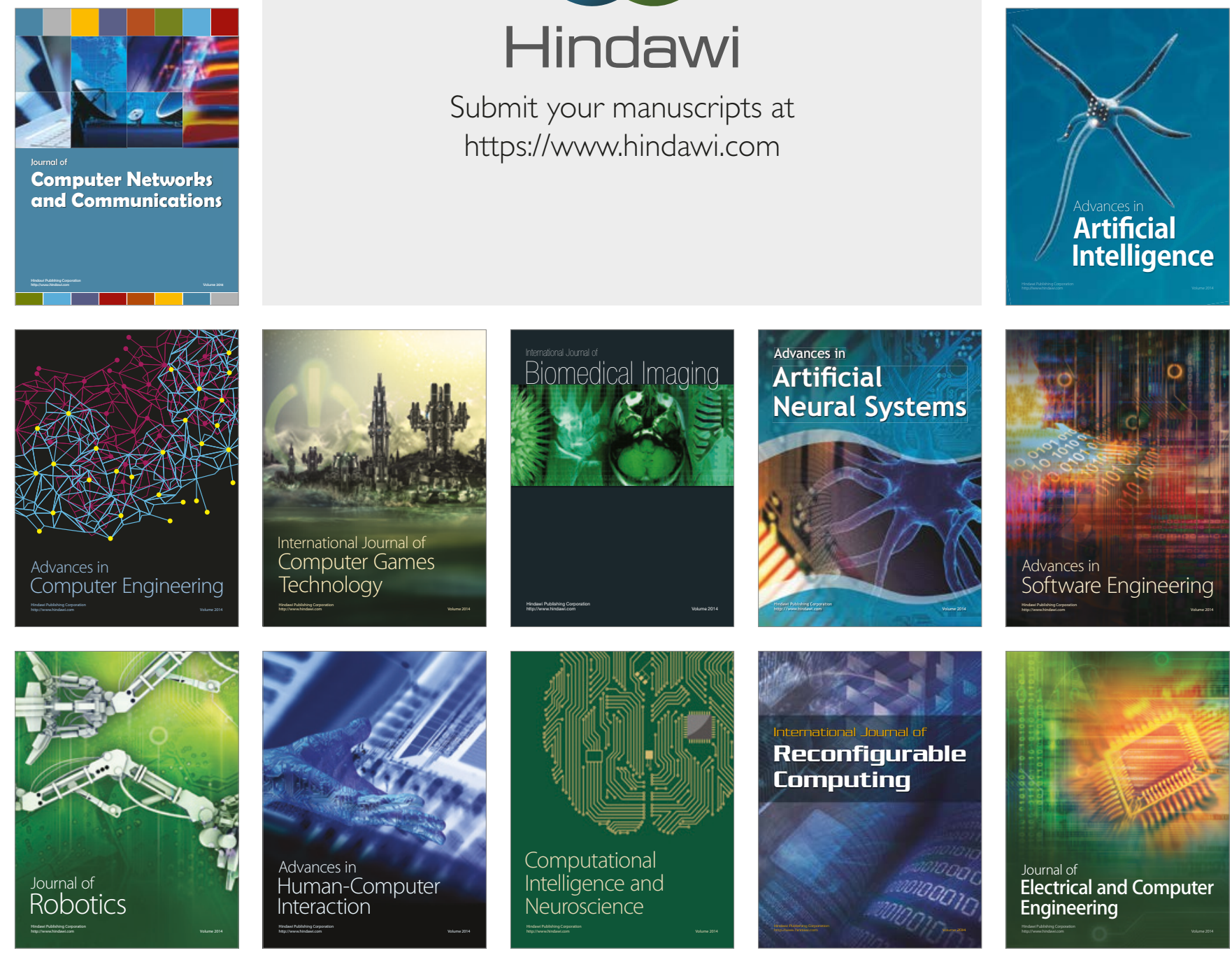